\title{
Clinicopathological Analysis of Anterior-Superior Mediastinal Masses
}

\author{
Sarah Grace Priyadarshini* and Ezhilvizhi Alavandar \\ Histopathology Department, Vijaya Hospital, Chennai
}

\begin{abstract}
Background: The mediastinum is the space between the two lungs, bounded anteriorly by the sternum and posteriorly by the vertebral column. A spectrum of neoplastic and non-neoplastic lesions can arise in the mediastinum.

Methods: A total of 14 cases were retrospectively retrieved from the Histopathology department. The cases had histopathological diagnosis either in image guided, thorocoscopic, transbronchial or excision biopsies.

Results: The majority of cases $(43 \% \mathrm{n}=6)$ were of thymic origin, which included both neoplastic lesions of thymoma group and nonneoplastic lesion like thymic cysts. The thymoma group included 3 cases of thymoma B 3 and 1 case of thymoma AB. The lymphoid lesions include Hodgkin lymphoma $(14.3 \% ; n=2)$ and non-Hodgkin lymphoma $(7.1 \% ; n=1)$. The mesenchymal lesions $(21.4 \%$; $=3)$ included lipoma, solitary fibrous tumor and schwannoma which accounts for one case each. A rare case of ectopic parathyroid adenoma $(7.1 \% ; n=1)$. and a case of metastatic papillary carcinoma $(7.1 \% ; n=1)$ were also reported.

Conclusion: The diagnosis of mediastinal mass lesions can pose difficulties due to its wide clinicopathologic spectrum to the clinician, radiologists and pathologist. A multimodal approach with histopathological examination and immunohistochemistry can help in arriving at an accurate diagnosis.
\end{abstract}

Keywords: Mediastinum, Thymoma, Lymphoma

\section{Introduction}

The mediastinum is the space in the thoracic cavity between the two lungs. It extends from the sternum anteriorly to the vertebral column posteriorly. It is divided anatomically into superior and inferior mediastinum. The inferior is further subdivided into anterior, middle and posterior mediastinum. The anterior mediastinum extends from T4 to T9 and is bound anteriorly by sternum, posteriorly by pericardium and laterally by pleural surfaces. This narrow space harbors the remnants of thymus and lymph nodes. Therefore, the most common neoplastic lesions in the anterior mediastinum are thymoma and lymphoma. Apart from these common neoplastic lesions a spectrum of benign and malignant mediastinal lesions has also been described in the anterior mediastinum. This study aims to characterize the histomorphologic spectrum of neoplastic and non-neoplastic lesions in the anterior mediastinum.

\section{Materials and Methods}

It is a descriptive retrospective study of cases of anteriorsuperior mediastinal masses. The study was done with full board approval of Institutional Ethics committee and approval for waiver of informed consent. The cases were retrieved from the histopathology department of a Superspeciality hospital from January 2018 to December 2019. A total of 14 cases of anterior-superior mediastinal masses were included in the study. The clinical presentation of all the patients were noted. The biopsy samples were processed in automated tissue processor, embedded in paraffin, cut into $5 \mu \mathrm{m}$ thick section and stained with Hematoxylin and eosin stain. The subsequent immunohistochemistry was done in a referral laboratory the results of which were recorded. Pan CK, LCA, CD3, CD20, CD5, CD117, EMA and Tdt are commonly used markers.

\section{Results}

The age of the patients ranged from 10 to 85 years with a mean age of 51 years. Out of 14 cases, 10 were males and rest were females with a male to female ratio of $2.5: 1$. The biopsies included excision biopsies $(n=8)$, CT guided biopsies $(n=4)$, thorocoscopic $(n=1)$ and transbrochial biopsies $(n=1)$.

The most common clinical presentation includes chest pain or discomfort $(78.6 \% ; n=11)$, cough $(64.3 \% ; n=9)$ and dyspnea $(42.9 \% ; n=6)$. One of the cases presented with muscle weakness and was treated as Myasthenia Gravis. On further evaluation was found to have an anterior mediastinal mass. The histopathology of the excision biopsy showed features of thymoma B3. The patient improved remarkably after surgery. One of patients with complaints of vague bone pains, on further evaluation showed hypercalcemia, 
mild hypophosphatemia and markedly elevated S.PTH $(1133 \mathrm{pg} / \mathrm{ml})$. The CT of this patient showed an anterior mediastinal mass. The histopathological of the excision showed features of parathyroid adenoma. In another patient with an initial presentation of chest discomfort and CT imaging revealed anterosuperior mediastinal cystic lesion. Image guided FNAC was done. The smears were paucicellular with few cyst macrophages. An excision biopsy was suggested. The histopathology was reported as metastatic papillary carcinoma to the mediastinum. The patient did not present with a thyroid lesion both clinically and in imaging, however a total thyroidectomy was done. Thorough examination of thyroid revealed a tiny focus of occult papillary carcinoma of thyroid in the isthmus.

CECT of chest was done in all the cases. All the cases of thymoma showed an enhancing homogenous mass lesion. One of the cases of Hodgkin lymphoma presented with a lobulated mildy enhancing mass lesion with projection into the lower portion of the right lobe of lung. The other case of Hodgkins presented with multiple mediastinal nodes. The patient with Non- Hodgkins lymphoma had a lobulated moderately enhancing lesion in the anterior mediastinum extending posteriorly and abutting the right main bronchus. All the mesenchymal lesions presented with non-enhancing homogenous mass lesions. Majority of them were solid lesions $(78.6 \% \mathrm{n}=11)$. The most common clinical and radiological differential diagnosis of anterior mediastinal solid tumors were lymphoma and thymoma. The cystic lesions accounted for $21.4 \%$ of cases $(n=3)$. This included the case of metastatic papillary carcinoma and two cases of thymic cyst in histopathology.

The majority of cases $(43 \% n=6)$ were of thymic origin, which included both neoplastic lesions of thymoma group $(28.7 \% \mathrm{n}=4)$ and non-neoplastic lesion like thymic cysts $(14.3 \% \mathrm{n}=2)$. The thymoma group included 3 cases of thymoma B3 and 1 case of thymoma AB (Fig1).

Table1:

\begin{tabular}{|l|l|}
\hline Clinical presentation & No of cases \\
\hline Chest discomfort & 11 \\
\hline Cough & 9 \\
\hline Dyspnoea & 6 \\
\hline Features of Myasthenia Gravis & 1 \\
\hline Features of hyperparathyroidism & 1 \\
\hline
\end{tabular}

Table 2:

\begin{tabular}{|l|l|}
\hline Histopathological Diagnosis & No of cases \\
\hline Thymoma & 4 \\
\hline Non-Hodgkin Lymphoma & 1 \\
\hline
\end{tabular}

Immunohistochemistry (IHC) was done in 2 cases of thymoma group. In the thymoma $\mathrm{AB}$ type the epithelial cells were positive for pan CK (Fig 2) and lymphoid cells were positive for CD3 and Tdt. It was negative for CD117. Histologically the possibilities of both thymoma types A and $\mathrm{AB}$ were suggested. Tdt positivity in IHC confirmed the diagnosis of type $\mathrm{AB}$ thymoma. One of the 3 cases of thymoma B3 showed atypical features like extensive invasion, necrosis and very scant lymphoid component. Immunohistochemistry revealed strong positivity for panCK and few scattered lymphoid cells showing Tdt expression. It was negative for CD117, CD5 and EMA. So it was diagnosed as an invasive thymoma B3. The other lymphoid lesions include Hodgkin lymphoma (14.3\% ; $\mathrm{n}=2)$ and non-hodgkin lymphoma $(7.1 \% ; \mathrm{n}=1)$. One of the cases of Hodgkin lymphoma showed predominantly spindle cells in a dense fibrocollagenous stroma and a focal collection of mixed inflammatory cells with lymphocytes and eosinophils. On careful examination few scattered Reed sternberg like cells were identified. The other histologic differential that was considered was an inflammatory myofibroblastic tumor. Immunohistochemistry was helpful in establishing the diagnosis of Hodgkin lymphoma. The transbronchial biopsy of one case showed sheets and nests of cells with high $\mathrm{N}$ :C ratio and vesicular nuclei and prominent nucleoli. The histological differential diagnosis of both poorly differentiated carcinoma and lymphoma were considered. The diagnosis of non-Hodgkin lymphoma Diffuse large B cell lymphoma (DLBCL) was confirmed by immunohistochemistry. It showed CD20 and MUM1 positivity with a high Ki67 (80\%). Therefore, IHC played a vital role in establishing the diagnosis mainly in cases of thymic and lymphoid lesions. The mesenchymal lesions $(21.4 \% ; n=3)$ included lipoma, solitary fibrous tumor and schwannoma which accounted for one case each. A rare case of ectopic parathyroid adenoma (Fig 3) and a case of metastatic papillary carcinoma were also reported. 


\begin{tabular}{|l|l|}
\hline Histopathological Diagnosis & No of cases \\
\hline Hodgkin Lymphoma & 2 \\
\hline Thymic cyst & 2 \\
\hline Lipoma & 1 \\
\hline Schwannoma & 1 \\
\hline Solitary fibrous tumor & 1 \\
\hline Metastatic papillary carcinoma & 1 \\
\hline Ectopic Parathyroid Adenoma & 1 \\
\hline
\end{tabular}

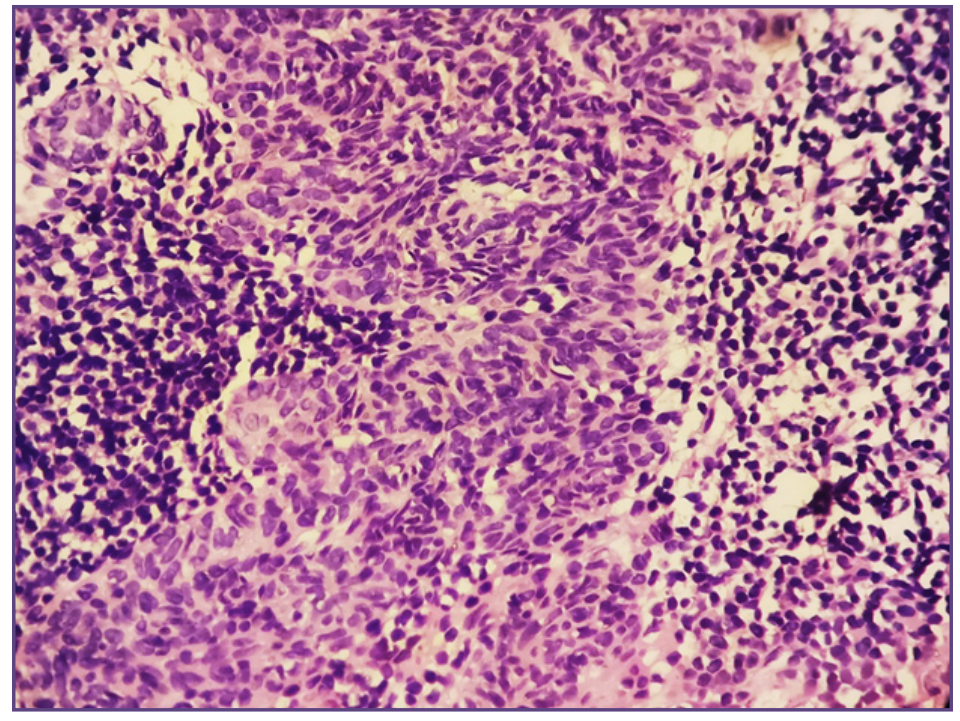

Fig. 1: A case of thymoma AB showing short fascicles of spindle cells and a discrete lymphocyte rich component (H\&E, 400X).

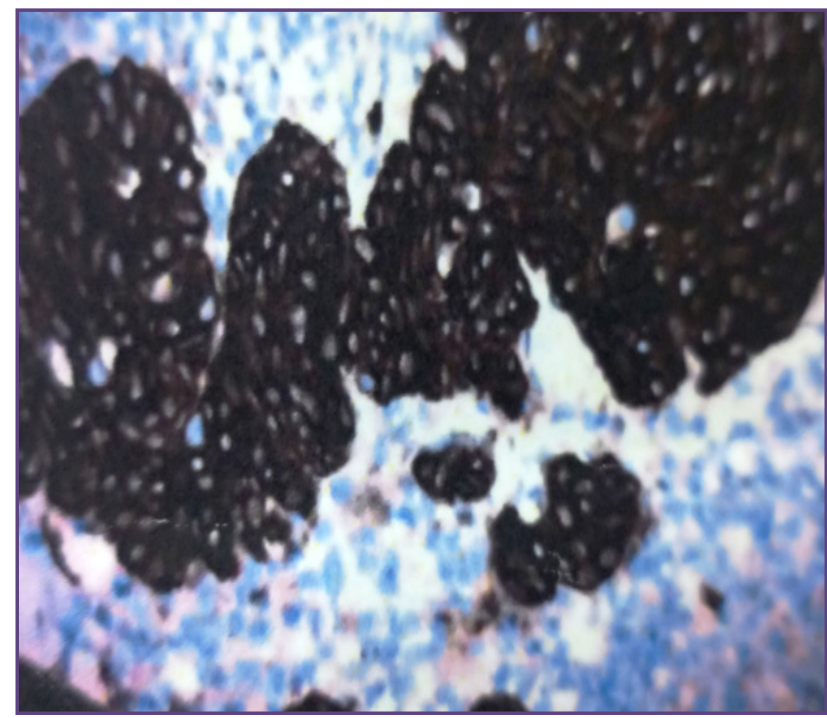

Fig. 2: Strong cytokeratin positivity of spindle cells in thymoma type AB (IHC - Pan CK), 400X).

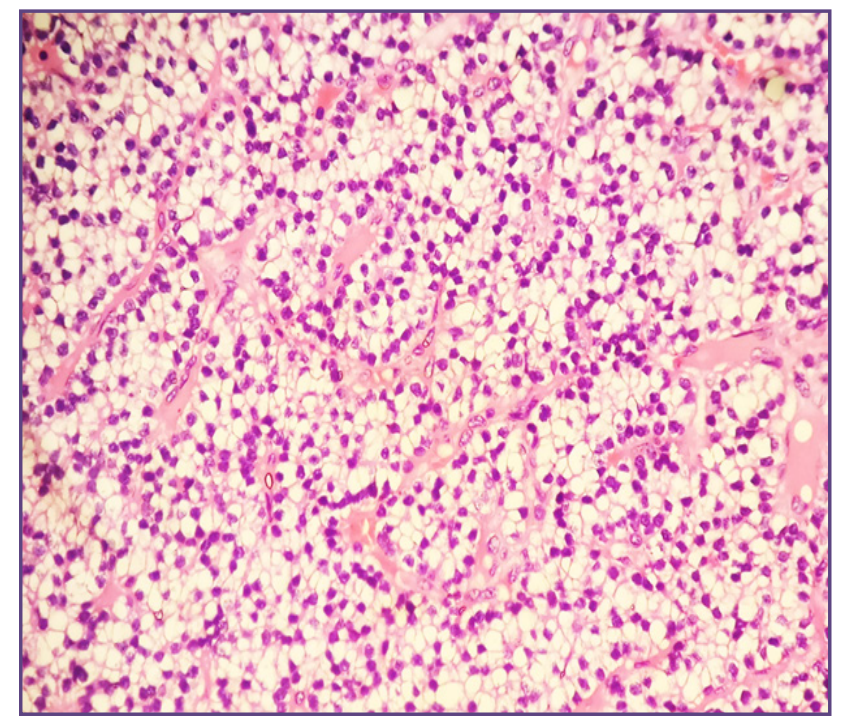

Fig. 3: A case ectopic parathyroid adenoma with polygonal cells with clear cytoplasm and regimentation of nuclei towards the vascular septae (H\&E, 400X). 


\section{Discussion}

The accurate diagnosis of mediastinal masses can be challenging both to the clinician and pathologist alike. The wide histopathologic spectrum, clinical heterogeneity, the difficulty in accessing this region especially in children because of its small area and presence of vital structures. However, with advent of image guided biopsy and use of immunohistochemistry (IHC), the diagnosis of these lesions has become more feasible ${ }^{[1]}$. Inspite of these advances the interpretation of mediastinal biopsies can be one of the most difficult ones for a pathologist. The smaller quantity of tissue, presence of crush artifact and the presence of histomorphological overlap of different entities can pose a serious problem ${ }^{[2]}$. The awareness of the different lesions that can arise in the mediastinum, correlation with imaging and use of ancillary techniques like IHC can help in arriving at a diagnosis, the accuracy of which becomes crucial in the selecting the right modality of treatment.

Most of the previous studies showed that the anterior mediastinal lesions were common in the fourth decade ${ }^{[1]}$. In the present study the mean age was 51 years slightly older than the previous studies. Patients presented with vague chest pain/discomfort, cough or dyspnea, evaluated using chest radiograph showed mediastinal widening. On the subsequent CT scan a diagnosis of mediastinal mass is confirmed ${ }^{[3]}$. Depending on the location a CT guided, transbronchial, thoracoscopic or excision biopsy was done.

The challenges in obtaining a diagnostic biopsy sample are primarily due to the difficulty in assessing the location and presence of vital structures. This results in various challenges to the pathologist owing to the smaller amount of tissue submitted for analysis and the presence of crush artifact making it difficult for the diagnosis to be made solely on histomorphology.

The lesions of thymic and lymphoid origin are the most common lesions in this location ${ }^{[4]}$. The distinction between them can pose a problem if the lymphoid component of a thymic neoplasm was only sampled, leading to erroneous diagnosis of a lymphoma. As these two entities have completely different treatment modalities the distinction becomes crucial. For this purpose, IHC plays a major role in highlighting the focal presence of epithelial component. Also, in the sub classification of thymic epithelial tumors, immunohistochemistry plays a major role ${ }^{[5]}$. The most useful markers are CD3, CD20, Tdt, Pan CK, EMA, CD5 and CD117.

The histomorphology of lymphoma can at times closely resemble a poorly differentiated carcinoma.
The differentiation between a metastatic carcinoma and lymphoma becomes vital in determining the prognosis and the suitable treatment modality for the patient. This distinction can also pose problems especially in small biopsies with extensive crush artifact resulting in pseudonesting or pseudo-trabecular pattern in a lymphoma leading to an erroneous diagnosis. In these situations, IHC with Pan CK and LCA plays a vital role for establishing a right diagnosis.

Apart from these common pathologic entities the rarer entities should also be kept in mind when examining a biopsy of a mediastinal lesion. In this study we had a case of an ectopic parathyroid adenoma of the anterior mediastinum. As the thymus and inferior parathyroid are derived from the same branchial pouch, embryologic remnants of parathyroid can be seen in the anterior mediastinum. Studies have shown that ectopic parathyroid is seen in $6 \%$ of healthy individuals and can give rise to the adenoma ${ }^{[6]}$.

Mediastinal cystic lesions are a heterogeneous group accounting for $18-25 \%$ of all mediastinal lesions ${ }^{[7]}$. In this study, $21.4 \%$ of cases showed a predominantly cystic lesion similar to the previous studies. Cystic lesions in the mediastinum can also pose a diagnostic difficulty. Image guided aspiration can be done, but it may not be possible to provide a diagnostic sample at all times. In such cases excision biopsy provides the final diagnosis. Though previous studies showed that most of the cystic lesions to be benign, we have seen in the present study a case of occult papillary carcinoma presenting with a cystic mediastinal metastatic lesion. The most common cystic lesion in the anterior mediastinum is a thymic cyst similar to that seen in the present study.

Mesenchymal tumors account for $2-6 \%$ of all mediastinal tumors ${ }^{[8]}$. In the present study the mesenchymal lesions were seen in $21.4 \%$ of cases, which is higher than the previous studies. The various mesenchymal lesions described in the anterior mediastinum include lipomatous tumors, solitary fibrous tumor, inflammatory myofibroblastic tumor, dendritic cell sarcoma, rhabdomyosarcoma and synovial sarcoma. Neural lesions are more common in the posterior mediastinum, but we had a case of schwannoma of anterior mediastinum in this study.

\section{Conclusion}

The difficulty in diagnosis of mediastinal lesions is attributed to its wide clinicopathologic spectrum, difficulty in assessing the lesions, smaller sample size, vast heterogeneity of lesion including both primary and 
metastatic. However, a multimodal approach with imaging, histopathological examination and the effective use of immunohistochemistry can help in arriving at an accurate diagnosis.

\section{Acknowledgements}

We would like to thank our technical staff Mrs Leyal, Mrs Manjula and Mrs Gauthami for their cooperation.

\section{Funding}

Nil

\section{Competing Interests}

Nil

\section{References}

1. Sharma P, JHA V, Kumar N, Kumar R, Mandal A. Clinicopathologic analysis of mediastinal masses: A mixed bag of non-neoplastic and neoplastic etiologies. Turk Patoloji Derg. 2017; 33: 37-46

2. Aggarwal R, Rao S, Chopra P, Bhalla S, Vijay C L, Bin Asaf B, and Kumar A. Morphological spectrum of mediastinal lesions with special emphasis on evaluation of needle biopsy: An experience from a tertiary care hospital. Indian J Med Res. 2016; 144(4): 544-551

3. Dalal U, Dalal A, Kartik A, Saini V, Anand L. Clinicopathological Profile of Primary Mediastinal Masses: Our Experience. J Surg Anesth 4:1. doi: 10.35248/26841606.20.4.126

4. Dasgupta S, Bose D, Bhattacharyya NK, Saha M, Biswas K, Biswas P K. A clinicopathological study of mediastinal masses operated in a tertiary care hospital in Eastern India in 3 years with special reference to thymoma. Indian J Pathol Microbiol . 2016;59 (1):20-24.

5. Marino M, Ascani S. An overview on the differential diagnostics of tumors of the anterior-superior mediastinum: the pathologist's perspective. Mediastinum 2019;3:6

6. Moran C, Suster S. Primary Parathyroid Tumors of the Mediastinum: A Clinicopathologic and Immunohistochemical Study of 17 Cases. Am J Clin Pathol 2005; 124:749-754

7. Petkar M, Vaideeswar P, Deshpande JR. Surgical Pathology of Cystic Lesions of The Mediastinum. Journal of Postgraduate Medicine 2001; 47 (4): 235-239

8. Bakker M D, Marx A, Strobel P. Mesenchymal tumours of mediastinum. Virchows Arch 2015; 467: 487-500

*Corresponding author:

Dr Sarah Grace Priyadarshini, Postal Address: C2 103 Akshaya Republic, Kundrathur main road, Kovur, Chennai 600122

Phone: 91-44-9790722496

Email: grace4ever03@gmail.com

Date of Submission $\quad: \quad 05 / 12 / 2020$

Date of Final Revision : 10/04/2021

Financial or other Competing Interests: None.

Date of Acceptance $\quad$ : $16 / 05 / 2021$

Date of Publication $\quad$ : $30 / 05 / 2021$ 